\section{Mobilität und Verkehr in der} Schweiz

\author{
Ansätze für eine umweltverträg- \\ lichere Gestaltung
}

\author{
von Ueli Balmer, Bundesamt für Raument- \\ wicklung, Schweiz
}

\begin{abstract}
Ein ausgezeichnetes Angebot im öffentlichen Verkehr, eine Güterverkehrspolitik mit klaren Zielen und Maßnahmen, hohe Standards bezüglich Umweltschutz, ein vergleichsweise flüssiger Individualverkehr diese und weitere Faktoren haben dazu geführt, dass die schweizerische Verkehrspolitik im Ausland vorwiegend positiv wahrgenommen wird. Auch wenn diese Wahrnehmung nicht in allen Teilen der Realität entspricht, hat die in den 1970er Jahren eingeleitete Verkehrspolitik doch auch mess- und spürbare Änderungen bewirkt, welche sie erklären und zumindest ansatzweise rechtfertigen. Die nachstehenden Ausführungen sollen dazu dienen, den Werdegang dieser Politik und damit mögliche Wege zu einer umweltverträglicheren Ausgestaltung des Verkehrs aufzuzeigen.
\end{abstract}

Wie ganz Westeuropa, war auch die Schweiz der Nachkriegsjahre geprägt von einer Aufbruchstimmung, welche sich im Verkehrsbereich in einer einseitig auf den motorisierten Individualverkehr ausgerichteten Investitionspolitik niederschlug. Beispielhaft dafür steht die Abstimmung über die Verbesserung des Straßennetzes, welche von der Schweizer Bevölkerung im Jahr 1958 mit einer erdrückenden Ja-Mehrheit von 85 Prozent angenommen wurde. ${ }^{1}$ Dieser Verfassungsartikel war die Grundlage für die Erstellung eines sehr großzügig ausgelegten Nationalstraßennetzes. Beim Schienenverkehr waren damals keine entsprechenden Verbesserungen vorgesehen.

\section{Die Gesamtverkehrskonzeption - ein kühner Wurf}

Die mit der Erstellung des Nationalstraßennetzes einhergehende, alle Erwartungen übertreffende Zunahme der Motorisierung zeigte die nachteiligen Konsequenzen dieser Politik bald auf: Während der öffentliche Verkehr zunehmend in die roten Zahlen „abrutschte“ und die öffentliche Hand entsprechend belastete, nahmen Raumbedarf und Umweltbelastung kontinuierlich zu. Diese beunruhigenden Entwicklungen veranlassten die Landesregierung, eine Expertenkommission mit der Erarbeitung einer Gesamtverkehrskonzeption (GVK) zu beauftragen. Im Rahmen dieser GVK sollten Mittel und Wege aufgezeigt werden, wie das schweizerische Verkehrssystem an die Anforderungen der Zukunft angepasst werden könnte. Als Hauptpunkte wurden

- die Beseitigung der Wettbewerbsverzerrungen im Rahmen einer freiheitlichen Verkehrspolitik,

- die Abgeltung der Auflagen der Verkehrsträger im Interesse der gesamten Volkswirtschaft und

- die Notwendigkeit der Koordination der Investitionen sowie das Erreichen der Eigenwirtschaftlichkeit der Verkehrsträger

bezeichnet. Diese Auftragsumschreibung macht deutlich, dass es bei der GVK nicht einfach um die Erarbeitung eines Verkehrswegeplanes oder eines Bauprogramms ging. Erwartet wurden vielmehr Vorschläge für eine Neuordnung der schweizerischen Verkehrspolitik.

Im Jahr 1977 präsentierte die Kommission das Ergebnis ihrer Arbeiten in Form von 40 konkreten Schlussempfehlungen. Sie lassen sich mit den vier folgenden Grundsätzen zusammenfassen:

1. Koordination der Planung, insbesondere koordinierter Ausbau der Verkehrsträger unter Einbezug der sozialen Kosten und Nutzen bei den Investitionsentscheiden

2. Zweckmäßige Aufgabenteilung zwischen Bund und Kantonen

3. Langfristig Eigenwirtschaftlichkeit der Verkehrsträger (mit voller Abgeltung gemeinwirtschaftlich erbrachter Leistungen und unter Abbau von Wettbewerbsverzerrungen),

4. Sicherstellung der Finanzierung auch des öffentlichen Verkehrs durch Steuer- und Zolleinnahmen des Bundes und Benutzungsabgaben der Unternehmungen. Der Finanzierungsmodus der Strasse mit Einnahmen aus Abgaben auf Treibstoffen sollte beibehalten und durch Benutzungsabgaben ergänzt werden. 
Gemessen an der bisherigen sektoralen Betrachtungsweise nahmen sich diese auf eine ganzheitliche Verkehrspolitik zielenden Forderungen der GVK geradezu kühn aus. Auf die anstehende politische Auseinandersetzung durfte man gespannt sein...

\section{Zu viele Jäger}

In der nachfolgenden politischen Diskussion wurde die Stoßrichtung der GVK zwar grundsätzlich begrüßt. Die zur Umsetzung erforderliche Vorlage geriet dann allerdings - ein bei ganzheitlichen Lösungsansätzen häufig auftretendes Phänomen - aus verschiedenen Gründen ins Wanken. Einen wichtigen Anlass dazu lieferte das eidgenössische Parlament, indem es zwei attraktive Elemente des Finanzierungsteils, die Schwerverkehrsabgabe und die Nationalstraßenabgabe, aus der Vorlage herausbrach und vorzeitig zur Abstimmung brachte. Während die Nationalstraßenabgabe nicht zuletzt zum Ziel hatte, die ausländischen Fahrzeuglenker an der Finanzierung des schweizerischen Straßennetzes zu beteiligen, sollten mit der Schwerverkehrsabgabe vor allem die ungedeckten Kosten dieser Fahrzeugkategorie gedeckt werden. 1984 wurden beide Vorlagen vom Volk angenommen; sie traten Anfang des darauf folgenden Jahres in Kraft. Die Schwerverkehrsabgabe wurde in Form einer Pauschale eingeführt und war ähnlich ausgestaltet wie die in verschiedenen EUMitgliedsländern noch heute zu entrichtende Eurovignette. Dabei war jedoch von Anfang an vorgesehen, die Abgabe zu einem späteren Zeitpunkt in eine leistungsabhängige Abgabe umzugestalten. Die rechtlichen Voraussetzungen für diese Umgestaltung sollten im Rahmen der Vorlage zur Umsetzung der GVK geschaffen werden.

Vier Jahre später (und elf Jahre nachdem die Expertenkommission ihre Vorschläge verabschiedet hatte) folgte die Abstimmung über diese Vorlage, die unter dem Kürzel „KVP“ bekannt wurde (Vorlage für eine koordinierte Verkehrspolitik). Allein schon diese lange Dauer lässt erahnen, wie heftig um deren Ausgestaltung gerungen worden war. Es war jedoch vergebliche „Liebesmüh“: Eine deutliche Mehrheit von 55 Prozent der Bevölkerung erteilte dem Paket eine Absage. Zentrale Argumente gegen die Neuregelung waren die Angst vor zu großer Einflussnahme des Bundes und die vorgeschlagene Möglichkeit zur Erhebung von Benutzungsgebühren, welche als Möglichkeit zur Erhebung neuer Steuern „gebrandmarkt" und deshalb insbesondere von den Straßenverkehrsverbänden vehement abgelehnt wurden.

Mit der Ablehnung der KVP-Vorlage waren die Probleme natürlich nicht gelöst. So fehlte die Grundlage für die vorgesehene spätere Umwandlung der „pauschalen“ in eine „leistungsabhängige“ Schwerverkehrsabgabe. Und unbeantwortet blieb auch die Frage, wie die geplante Modernisierung der EisenbahnInfrastruktur künftig finanziert werden sollte. Neue Dynamik erhielt die verkehrspolitische Diskussion durch die Entwicklung im Alpen querenden Straßengüterverkehr, welche eine umweltorientierte Güterverkehrspolitik in die Wege leitete.

\section{Güterverkehr - konsequent in Richtung Nachhaltigkeit}

\subsection{Brennpunkt Gotthard}

Über Jahrzehnte, genau genommen bis zur Eröffnung des Gotthard-Straßentunnels im Jahr 1980, hatte die Bahn im Gütertransport durch die (Schweizer) Alpen eine übermächtige Stellung. Der Anteil der Strasse bewegte sich im einstelligen Prozentbereich. Dies änderte sich mit der Eröffnung des Straßentunnels schlagartig. Von einigen Hundert stieg die Zahl schwerer Güterfahrzeuge innerhalb von 20 Jahren auf täglich rund 4.000 Fahrzeuge an. Die damit verbundenen Nebenwirkungen in Form von Lärm und Schadstoffen, welche durch die topographischen Verhältnisse im Gebirge drastisch verstärkt wurden, führten $\mathrm{zu}$ vehementen Reaktionen bei der betroffenen Bevölkerung. Deren Unmut entlud sich in der Lancierung einer Volksinitiative zum Schutz des Alpengebietes. Kernpunkte dieser Volksinitiative waren die Forderungen nach einer vollständigen Verlagerung des Alpen querenden Transitgüterverkehrs auf die Schiene und der Verzicht auf die Erweiterung der Straßenkapazität. Dank einer starken Solidari- 
sierung der nicht direkt betroffenen Stimmbürgerinnen und Stimmbürger mit den Anliegen der Bergbevölkerung fand die Initiative im Jahr 1994, gegen den Willen der Landesregierung und des Parlaments, eine Mehrheit im Volk. Eine wesentliche Rolle spielte dabei der zwei Jahre zuvor gefasste Entscheid zum Bau zweier neuer Bahntunnel durch die Alpen, der mit der Notwendigkeit der Verkehrsverlagerung begründet worden war. Vielen der Abstimmenden schien die Forderung nach einer konsequenten Verlagerung des Transitgüterverkehrs auf die Schiene nichts weiter als die logische Fortsetzung der eingeleiteten Politik.

Mit der zwingenden Verpflichtung, den Transitgüterverkehr auf die Schiene zu verlagern, geriet die Schweiz international in eine schwierige Lage. Schließlich betraf diese Vorgabe doch fast ausschließlich ausländische Fahrzeuge und wurde dementsprechend im Ausland als diskriminierend wahrgenommen. Dies geschah zu einem Zeitpunkt, als die Schweizer Wirtschaft eigentlich bestrebt war, die Nachteile des „Abseitsstehens“ von der europäischen Union und dem europäischen Wirtschaftsraum zu überwinden. ${ }^{2}$ Dementsprechend galt es, eine Lösung zu finden, welche den unterschiedlichen Anliegen der involvierten Kreise Rechnung trug:

- dem Begehren der EU nach möglichst ungehinderter Durchfahrt des Güterverkehrs durch die Schweiz und dabei insbesondere der Anhebung der in der Schweiz noch geltenden Gewichtslimite von 28 auf 40 Tonnen,

- dem Bedürfnis der Bergbevölkerung nach Schutz vor dem Transitverkehr und

- den Interessen der Schweizer Wirtschaft nach möglichst ungehinderten Handelsbeziehungen zur EU resp. den Nachbarstaaten.

\subsection{Joker „Leistungsabhängige Schwerverkehrsabgabe“}

Bei der Suche nach einer allseits akzeptablen Lösung rückte die Umwandlung der Schwerverkehrspauschalen in eine leistungsabhängige Abgabe (LSVA) immer mehr in den Vordergrund. Die Verteuerung des Straßengüterverkehrs, die über diese Abgabe möglich wurde, lieferte eine marktkonforme und diskriminierungsfreie Grundlage für die angestrebte
Verlagerung auf die Schiene, trug damit den Schutzinteressen der Bergbevölkerung Rechnung und ermöglichte gleichzeitig den Abschluss der von der Schweizer Wirtschaft gewünschten bilateralen Verträge. Denn es war von vornherein klar, dass das Stimmvolk dem Abschluss solcher Verträge nur zustimmen würde, wenn darin auch die zum Schutz des Alpengebietes erforderlichen Maßnahmen festgeschrieben würden. Dank des Einbezugs der „externen Kosten“ in die Berechnung der Schwerverkehrsabgabe erreichte diese ein Niveau, das eine nachhaltige Wirkung auf das Verkehrsgeschehen erwarten lassen konnte. Dies war nicht zuletzt deshalb wichtig, weil die von der EU verlangte Zulassung von 40tFahrzeugen auf dem schweizerischen Straßennetz den Straßentransport tendenziell verbilligte. Tatsächlich liegt denn auch der Satz der Schwerverkehrsabgabe in der Schweiz mit rund 65 Eurocent pro Kilometer (für ein 40tFahrzeug) deutlich höher als in Deutschland (mit 12,4 €Ct.) und Österreich (ca. 22 €Ct.).

Nach einem äußerst kontrovers geführten Abstimmungskampf stimmte das Volk 1998 der Umwandlung der pauschalen in eine leistungsabhängige Schwerverkehrsabgabe $\mathrm{zu}$. Parallel dazu wurde entschieden, zwei Drittel des Abgabeertrages zur Finanzierung großer Vorhaben des öffentlichen Verkehrs, insbesondere der Realisierung neuer Bahntunnel durch die Alpen, zu verwenden. Das restliche Drittel geht an die Kantone. Im Bewusstsein, dass mit der Schwerverkehrsabgabe allein die angestrebte zusätzliche Verlagerung auf die Bahn nicht zu schaffen sein würde, wurden mehrere weitere Maßnahmen in die Wege geleitet. Dazu gehören der Bau neuer Eisenbahn-Alpentransversalen durch das Gotthardmassiv und den Lötschberg, eine Bahnreform, um dieses Verkehrsunternehmen leistungs- und damit wettbewerbsfähiger zu machen, sowie verstärkte Kontrollen auf der Strasse zur Durchsetzung der geltenden gesetzlichen Bestimmungen.

\subsection{Positive Bilanz}

Vor gut fünf Jahren, am 1. Januar 2001 trat die leistungsabhängige Schwerverkehrsabgabe in Kraft. Der Einführungstarif wurde auf 1,6 Rappen pro Tonnenkilometer festgelegt. Im 
Jahr 2005 wurde dieser Satz um rund 50 Prozent auf 2,4 Rappen (rund 1,6 €Ct.) erhöht. Bei der Analyse der seither eingetretenen Entwicklung ist $\mathrm{zu}$ berücksichtigen, dass die Schweiz (wie mit der EU vereinbart) die Gewichtslimite schrittweise auf die in Westeuropa üblichen 40 Tonnen anhob. Diese Maßnahme ermöglichte dem Straßentransport die Realisierung von Produktivitätsgewinnen, welche die Mehrbelastung durch die LSVA weitgehend kompensierte. Vor diesem Hintergrund ist es nicht erstaunlich, wenn der Regimewechsel weniger $\mathrm{zu}$ einer Verlagerung auf die Bahn als mehr zu einer deutlichen Produktivitätssteigerung im Straßentransport führte. Die nachstehende Abbildung 1 veranschaulicht die positiven Auswirkungen der ergriffenen Maßnahmen auf das Verkehrsaufkommen: In den zwei Jahren nach dem Regimewechsel sank die Fahrleistung der von der Abgabe erfassten Fahrzeuge um rund zehn Prozent und lag Ende 2004 immer noch sieben Prozent unter dem Niveau des Jahres 2000. Im Rahmen einer wissenschaftlichen Studie, die von der Bundesverwaltung in Auftrag gegeben worden war, wurde untersucht, wie weit der festgestellte Fahrleistungsrückgang tatsächlich auf die leistungsabhängige Schwerverkehrsabgabe zurückzuführen sei. Als erstes widerlegten die Autoren der Untersuchung die gelegentlich gehörte Behauptung, dass vermehrt Lieferwagen anstelle abgabepflichtiger LKWs eingesetzt würden. ${ }^{3}$ Einen gewissen Einfluss hatte demgegenüber der schleppende Konjunkturverlauf; ihm konnten 20 Prozent der eingetretenen Wirkung zugeschrieben werden. Die verbleibenden 80 Prozent verteilten sich zu praktisch gleichen Teilen auf die neue Abgabe und die höhere Gewichtslimite.

Noch deutlichere Spuren hinterließ das neue Regime im Bereich des Alpen querenden Güterverkehrs. In diesem Bereich sank die Zahl der Fahrten seit Einführung des neuen Regimes um rund 15 Prozent (siehe Abb. 1). Für das Jahr 2006 zeichnet sich eine Fortsetzung dieser angestrebten Entwicklung ab. Als besonders erfreulich ist die Tatsache zu bezeichnen, dass die Bahnen ihren Anteil am Verkehrsaufkommen, welcher infolge der Einführung der höheren Gewichtslimite zwischenzeitlich gesunken war, inzwischen wieder auf rund zwei Drittel zu steigern vermoch- ten. Die Entwicklung zeigt, dass die angestrebte Verlagerung auf die Bahn bei einer konsequenten Politik möglich ist. Mit der weiteren Umsetzung der Bahnreform und der Eröffnung der Bahntunnel durch den Lötschberg (im Jahr 2007) und durch das Gotthardmassiv (voraussichtlich im Jahr 2016) sind die Rahmenbedingungen für eine weitere positive Entwicklung im Güterverkehr gesetzt.

Abb. 1: Gefahrene Fahrzeugkilometer vor dem Hintergrund der Leistungsabhängigen Schwerverkehrsabgabe im Alpen querenden Verkehr (1997-2004)

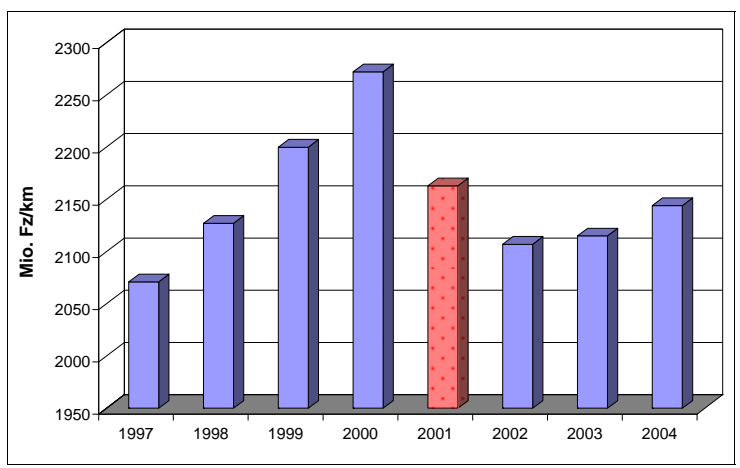

Datengrundlage: Bundesamt für Verkehr, Alpinfo

\section{Ansätze beim Personenverkehr}

In der Schweiz kommen auf einen Lkw rund 40 Pkw. Auch wenn ein Güterfahrzeug natürlich wesentlich mehr Raum beansprucht als ein Personenwagen, macht der Vergleich doch deutlich, dass eine noch so konsequente Güterverkehrspolitik die Probleme auf den Strassen nicht zu lösen vermag. Eine Grundvoraussetzung, um die sich stellenden Probleme erfolgreich lösen zu können, ist ein gutes Angebot im öffentlichen Verkehr. Mit dem Projekt „Bahn 2000“ beschritt die Schweiz auch in diesem Bereich einen eigenen Weg.

\subsection{Das Konzept „Bahn 2000“}

„Nicht so schnell wie möglich, sondern so schnell wie nötig!“ Nach diesem Motto sollte im Rahmen des Konzeptes „Bahn 2000“ die Schieneninfrastruktur so erneuert werden, dass die Fahrzeiten zwischen den größeren Städten des Landes auf eine knappe Stunde 
reduziert und damit optimale Umsteigebeziehungen sowohl zu den andern IC-Verbindungen als auch zum regionalen Verkehr auf Strasse und Schiene gewährleistet werden können. Der konsequente Einbezug des regionalen Verkehrs auf Schiene und Strasse war eine zentrale Voraussetzung, um dieses Konzept mehrheitsfähig zu machen. Das Vorläuferprojekt „Neue helvetische Transversale“ war wegen seiner einseitigen Ausrichtung auf die großen Zentren auf entsprechende Kritik gestoßen. Rückgrat des Konzepts „Bahn 2000“ bildet eine Neubaustrecke im Raum zwischen Olten und Bern, welche die angestrebten Fahrzeitreduktionen zwischen Bern einerseits sowie Basel, Zürich und Luzern andererseits ermöglicht. Im Dezember 2004, also knapp fünf Jahre später als ursprünglich vorgesehen, folgte mit der Inbetriebnahme der Neubaustrecke der wichtigste Schritt im Rahmen der Realisierung. Obwohl die Neubaustrecke infolge Schwierigkeiten bei der Einführung des neuen Sicherheitssystems ETCS $^{4}$ vorläufig nicht wie geplant mit 200, sondern nur mit 160 Stundenkilometern befahren werden kann, hat sich Bahn 2000 in der Praxis gut eingespielt und es den Bahnen er- laubt, ihre aus dem Autobahnbau resultierenden Konkurrenznachteile gegenüber der Strasse teilweise aufzuholen (siehe Abb. 2). Im Rahmen des Projekts „Zukünftige Entwicklung der Bahninfrastruktur" soll die Grundidee von Bahn 2000 weitergeführt werden. Mit den zur Verfügung stehenden Mitteln in der Größenordnung von sechs bis sieben Milliarden Schweizer Franken soll die Schieneninfrastruktur im Zeitraum 2013 bis 2030 gezielt weiter ausgebaut werden. Dies ermöglicht einerseits eine Senkung der Reisezeiten auf der wichtigen West-Ost Achse um eine halbe Stunde und andererseits die Einbindung weiterer Städte in das Konzept von Bahn 2000. Im Bereich Güterverkehr stehen ebenso Verbesserungen der Produktivität im Vordergrund.

\subsection{Der Infrastrukturfonds - ein Ansatz für die Agglomerationen}

Mit den eingeleiteten Maßnahmen zugunsten des öffentlichen Verkehrs ist es zwar gelungen, dessen Anteil am gesamten Verkehrsaufkommen nicht nur zu halten, sondern sogar wieder leicht zu steigern. Den Wachstums-

Abb. 2: Schematische Darstellung „Bahn 2000“

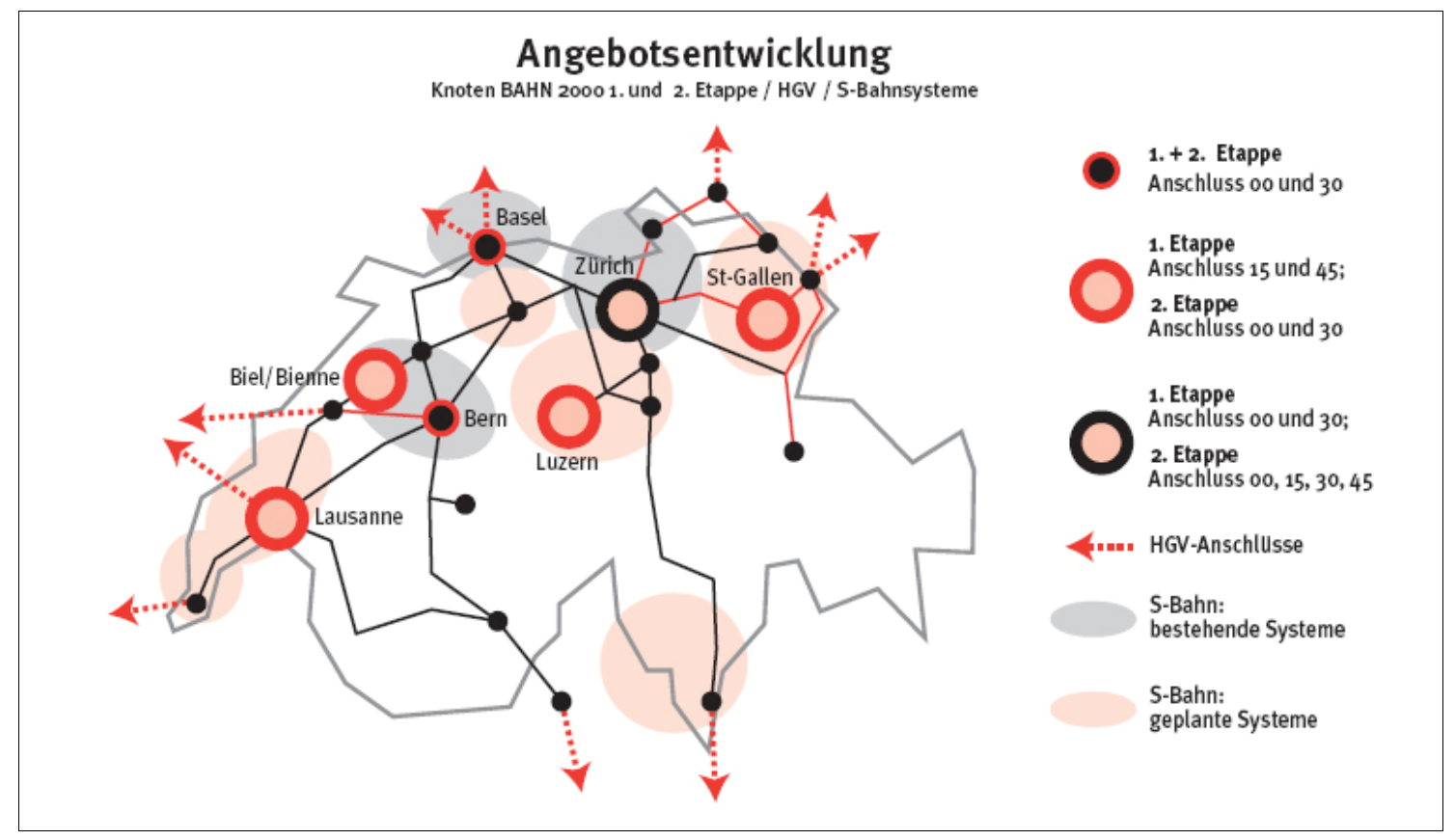


trend des motorisierten Individualverkehrs vermochte dies allerdings nicht zu brechen. Inzwischen ist dieser so weit angewachsen, dass auch in der Schweiz Staus zunehmend zum Alltag gehören. Besonders nachdrücklich zeigt sich dies in den Agglomerationen. Gemäß Auswertungen des Bundesamtes für Raumentwicklung entstehen in diesen Räumen 85 bis 90 Prozent aller Staus in der Schweiz. Die Staus, die außerhalb der Agglomerationen auftreten, sind zum größten Teil auf saisonale, d. h. nicht tägliche oder wöchentlich wiederkehrende Überlastungen zurückzuführen. Die Lösung der anstehenden Probleme wird erschwert durch den Umstand, dass die Erträge aus den Verkehrsabgaben (Motorfahrzeugsteuern, Nationalstraßenabgabe, LSVA und Mineralölsteuern) fast ausschließlich Bund und Kantonen zukommen, während die von den Staus direkt betroffenen Gemeinden für die Lösung ihrer Verkehrsprobleme auf allgemeine Finanzierungsmittel zurückgreifen müssen. Vor diesem Hintergrund ist die vom Parlament kürzlich verabschiedete Vorlage für die Schaffung eines Infrastrukturfonds zu sehen.

Konkreter Auslöser dieser Vorlage zur Schaffung eines Infrastrukturfonds war die deutliche Verwerfung einer einseitig am Straßenbau orientierten Abstimmungsvorlage durch das Schweizer Stimmvolk. Die Vorlage übernimmt das Grundanliegen dieses abgelehnten Begehrens, das die Verflüssigung des zunehmend ins Stocken geratenden Verkehrs formulierte, zielt jedoch auf eine gesamtheitliche Lösung. Danach werden zwar Mittel zur Mitfinanzierung von Verkehrsprojekten in Agglomerationen bereitgestellt, Beiträge werden jedoch nur gewährt, wenn die Antrag stellenden Körperschaften (Kantone, allenfalls Gemeindeverbände) ein Agglomerationsprogramm erarbeitet haben, welches ein ganzheitliches Vorgehen garantiert. Dazu gehört ein zweckmäßiger Einbezug der verschiedenen Verkehrsträger unter Berücksichtigung der angestrebten Siedlungsentwicklung. Wie der Name der Vorlage („Bundesgesetz über den Infrastrukturfonds für den Agglomerationsverkehr und das Nationalstraßennetz") besagt, beschränkt sich deren Lösungsansatz nicht auf den Agglomerationsverkehr. Vielmehr werden auch Mittel zur Beseitigung von Engpässen auf dem Nationalstraßennetz zur Verfügung gestellt. Alimentiert werden soll der Fonds aus Erträgen von Straßenverkehrsabgaben.

\subsection{Steigerung der Benutzerfreundlichkeit}

Eine gute Infrastruktur und ein dichtes Angebot kommen nur dann effektiv zum Tragen, wenn auch deren Benutzung attraktiv gestaltet wird. Auch in diesem Bereich wurden in den letzten Jahrzehnten erhebliche Fortschritte erzielt. Einen wichtigen Meilenstein stellten in diesem Zusammenhang die regionalen Umweltabonnements dar, die im Gefolge der Diskussion über das Waldsterben in den 1980er Jahren eingeführt wurden. Diese kostengünstig erhältlichen Abonnements ermöglichen die beliebige Benutzung des öffentlichen Verkehrs innerhalb einer Region. Parallel dazu wurde der Geltungsbereich des großen Bruders dieser Umweltabonnements, des Generalabonnements, zunehmend ausgedehnt. Es ermöglicht heute freie Fahrt auf dem Netz der meisten Bahn-, Schifffahrts- und öffentlichen Busbetrieb, insbesondere auch denjenigen des Nahverkehrs. Zudem werden im touristischen Bereich bei Vorweisen des Generalabonnements häufig Ermäßigungen gewährt. Nur gelegentliche Benützer des öffentlichen Verkehrs haben die Möglichkeit, durch Erwerb eines kostengünstigen „Halbtax-Abonnements“ ihre Fahrtkosten im Einzelfall zu halbieren. Die große Popularität und starke Benutzung des öffentlichen Verkehrs in der Schweiz hängen sicher auch mit diesen attraktiven Angeboten zusammen.

\section{Planerische Instrumente}

Auf der planerischen Ebene stehen verschiedene Instrumente zur Verfügung, welche auch im Dienste der Nachhaltigkeit stehen. Nachstehend seien zwei innovative Elemente kurz dargestellt:

- Das Projekt NISTRA („Nachhaltigkeits-Indikatoren für Straßeninfrastrukturprojekte"), das vom zuständigen Bundesamt für Strassen bei größeren Bau- und Ausbauprojekten von National- und Hauptstrassen angewendet wird, liefert den Entscheidungs- 
trägern Beurteilungsgrundlagen, welche auf dem Konzept der Nachhaltigkeit beruhen und den politischen Abwägungsprozess vereinfachen. Im Rahmen von NISTRA wird ein Projekt anhand von 40 Indikatoren aus den Bereichen „Umwelt“, „Wirtschaft“ und „Gesellschaft“ "bewertet. Diese Indikatoren werden zum Teil monetarisiert, zum Teil quantifiziert (in Punkten) und zum Teil qualitativ (in Worten) dargestellt. Für die Entscheidungsfindung werden die verschiedenen Teilergebnisse bewusst nicht zu einer einzigen Maßzahl verdichtet, sondern in einer Übersicht transparent gemacht.

- Mit dem „Leitbild Verkehrstelematik“ wird aufgezeigt, was Telematikanwendungen zur Erreichung der verkehrspolitischen Ziele beitragen können. Es konzentriert sich auf den Bereich Strasse, da hier der größte Handlungsbedarf besteht. Es zeigt aber auch die Schnittstellen zum öffentlichen Verkehr auf und trägt dem Gedanken der koordinierten Verkehrspolitik Rechnung. $\mathrm{Zu}$ seiner Umsetzung bedarf es spezifischer Instrumente (u. a. des Projekts „Verkehrsmanagement Schweiz") $)^{5}$.

Die Implementierung der Projekte NISTRA und „Leitbild Verkehrstelematik“ entspricht der Forderung nach sorgfältigen, den Prinzipien der Nachhaltigkeit entsprechenden Planungsentscheiden, die schon von der Gesamtverkehrskommission erhoben wurde (zur Gesamtverkehrskommission siehe oben).“ Diese Implementierungen stellen damit einen weiteren Mosaikstein im Rahmen der punktuellen Umsetzung der Schlussempfehlungen dieser Kommission dar.

\section{Rück- und Ausblick}

Als die Arbeiten an der Gesamtverkehrskonzeption aufgenommen wurden, steckte die Umweltdiskussion noch in den Anfängen. Dessen ungeachtet haben die Überlegungen, die im Rahmen dieser Arbeiten angestellt wurden, die hier vorgestellten Ansätze in Richtung auf eine umweltorientierte Verkehrspolitik wesentlich beeinflusst. Beispiele dafür sind die mit der LSVA erfolgte Umsetzung des „Prinzips Kostenwahrheit“ im Bereich Straßengüterverkehr, der gezielte Aus- bau des öffentlichen Verkehrs und die Querfinanzierung Schiene / Strasse. Jüngstes Beispiel für das Angehen einer schon im Rahmen der GVK erkannten Problematik des unkoordinierten Ausbaus der Verkehrsträger stellt der Gesetzesentwurf für einen Infrastrukturfonds dar, der sich in der parlamentarischen Beratung befindet. Unter Voraussetzung einer Einbettung in ein Gesamtkonzept (Agglomerationsprogramm) wird er finanzielle Beiträge des Bundes an Infrastrukturprojekte in den von der Verkehrsentwicklung buchstäblich überrollten Agglomerationen ermöglichen.

Bezüglich einer Lenkung über den Preis im Bereich Personenverkehr üben sich der Bundesrat (die Landesregierung) und das Parlament in Zurückhaltung. In Beantwortung eines parlamentarischen Vorstoßes hat der Bundesrat den Rahmen wie folgt gesetzt:

\begin{abstract}
„Das so genannte ,Road-Pricing’ ist mit einem mittel- bis langfristigen Realisierungshorizont ein zukunftsträchtiger und interessanter Ansatz. Es erlaubt eine verursachergerechte Verkehrslenkung nach verschiedenen Faktoren (z. B. Zeit, Ort, gefahrene Kilometer, Stauverhältnisse). Beispiele aus dem Ausland und die leistungsabhängige Schwerverkehrsabgabe in der Schweiz zeigen, dass solche Gebühren heute technisch und betrieblich machbar sind und bei guter Ausgestaltung auch auf politische Akzeptanz stoßen können. Angesichts der großen Verkehrsprobleme und der fehlenden Mittel für die Modernisierung der Verkehrsinfrastrukturen dürften mittelfristig Road-Pricing Lösungen für Städte und Agglomerationen im Vordergrund stehen. Längerfristig könnte auch ein flächendeckendes Road-Pricing für die ganze Schweiz - ggf. als teilweiser Ersatz der heutigen Verkehrsabgaben - in Betracht gezogen werden. In diesem Fall müsste das ganze System der Verkehrsfinanzierung überprüft und sowohl in technischer Hinsicht als auch bezüglich der Höhe der Verkehrsabgaben auf die Situation im Ausland abgestimmt werden.“ (Interpellation Walker 2004)
\end{abstract}

Das Parlament hat seinerseits von der Regierung verlangt, in einem Bericht darzulegen, unter welchen Bedingungen und in welcher Form die Einführung eines Road-Pricings in der Schweiz sinnvoll wäre. Insbesondere solle der Bericht darüber Auskunft geben, 
a) ob die Einführung des Road-Pricings eine Änderung der Bundesverfassung voraussetze (Gemäß geltender Verfassung ist die Straßenbenützung grundsätzlich gebührenfrei.);

b) für welche Strassen, zu welchen Zwecken und mit welchen Bemessungsgrundlagen ein Road-Pricing allenfalls sinnvoll wäre und wie allenfalls Kompensationen bei anderen Verkehrsabgaben erfolgen könnten;

c) unter welchen Bedingungen allenfalls ein Versuch mit Road-Pricing sinnvoll und möglich wäre.

Parallel zur laufenden Erarbeitung dieses Berichts wurde ein Forschungspaket „Mobility Pricing“ lanciert. Im Rahmen dieses Projektes sollen für die Schweiz relevante Wissenslücken zur Anwendbarkeit von Preiselementen für die Benützung von Verkehrsanlagen geschlossen werden. Die zum Teil heftigen Reaktionen auf dieses Projekt und auf - seien sie auch noch so vage gehalten - politische Absichtserklärungen zum Thema Road-Pricing zeigen auf, wie sensibel dieser Bereich ist. Auf die Fortsetzung dieser Geschichte darf man sehr gespannt sein.

\section{Anmerkungen}

1) Für Erläuterungen zu den Besonderheiten des schweizerischen Politsystems vgl.: http://www. bk.admin.ch/themen/pore/?lang=de

2) In einer denkwürdigen Abstimmung hatte das Schweizer Stimmvolk den Beitritt zum Europäischen Wirtschaftsraum im Jahr 1992 abgelehnt.

3) Bundesamt für Raumentwicklung: Entwicklung des Straßengüterverkehrs, S. $30 \mathrm{ff}$.

4) ETCS steht für „European Train Control System".

5) Unter Verkehrsmanagement wird die Verkehrsbeeinflussung mit den Hauptfunktionen Verkehrslenkung, -leitung und -steuerung sowie das Management des ruhenden Verkehrs und der Fahrtberechtigung verstanden.

\section{Literatur}

Bundesamt für Raumentwicklung, 2004: Entwicklung des Strassengüterverkehrs nach Einführung von LSVA und 34t-Limite; http://www.are.admin. ch/are/de/verkehr/lsva/index.html / 23.11.2006

Bundesamt für Verkehr; Alpinfo, 2005: http://www.bav.admin.ch/themen/verkehrspolitik/00 501/index.html?lang=de / 23.11.2006

Interpellation Walker Felix, 2004: Verkehr. Finanzpolitische Standortbestimmung vom 16. März 2004; http://search.parlament.ch/cv-geschaefte? gesch_id=20043075 / 23.11.2006

\section{Kontakt}

Ueli Balmer

Stellvertretender Leiter Sektion Verkehrspolitik Bundesamt für Raumentwicklung ARE

3003 Bern, Schweiz

Tel: +41 - 31 - 3249735

E-Mail: ueli.balmer@are.admin.ch

\section{《》}

